\title{
Construção de Controlador Lógico Programável de Baixo Custo para Fins Didáticos
}

\author{
Construction of Low-Cost Programmable Logic Controller for Educational Purposes
}

\section{Yuri Barros de Miranda Neves ${ }^{1}$ (Dorcid.org/0000-0002-8722-3498}

\section{Diogo Roberto Raposo de Freitas ${ }^{1}$ (Dorcid.org/0000-0002-5023-699X}

${ }^{1}$ Escola Politécnica de Pernambuco, Universidade de Pernambuco, Recife, Brasil.

E-mail do autor principal: Yuri Barros de Miranda Neves yuribmneves@gmail.com

\section{RESUMO}

Montar laboratórios de automação industrial com aparatos de práticas com controladores lógicos programáveis comerciaissão custosos para Universidades Públicas de Ensino. A proposta deste trabalho é criar um controlador lógico programável de baixo custo para aplicações didáticas. Foi feito o circuito eletrônico e utilizado softwares de uso livre Ladder Maker e Arduino. O circuito eletrônico foi planejado com componentes de baixo valor de compra no mercado. A confiabilidade dos componentes eletrônicos utilizados não foi de análise criteriosa para que o custo de fabricação não elevasse. Foi realizada uma comparação do custo total do projeto do CLP de baixo custo com o custo de aquisição de CLPs à venda no mercado e foi possível comprovar que o custo fica em torno de $15 \%$ comparado aos equipamentos de empresas tradicionais. A vantagem do valor de confecção deste CLP se sobressai mesmo com menor confiabilidade, visto que a ideia é para fins didáticos.

PALAVRAS-CHAVE: Controlador lógico programável; Ensino de Engenharias; Aplicações Didáticas.

\section{ABSTRACT}

Assemblingindustrial automation laboratories with practical apparatus with commercial Programmable Logical Controllers (PLC)iscostly for Brazilian public universities. The proposal of this paper is to create a programmable logic controller for didactic applications. It was made the electronic circuit and used free-software Ladder Maker and Arduino. The electronic circuit was designed with low-cost components in the market. The reliability of the electronic components used was not of careful analysis so that the cost of manufacture did not rise. A cost comparison was made between the proposed PLC project and purchasing PLCS sold in the market and it was possible to show that the cost is around $15 \%$. The advantage of the manufacturing value of this $P L C$ stands out even with less reliability since the idea is for didactic purposes.

KEY-WORDS: Programmable Logic Controller; Engineering Education; Didactic Applications. 


\section{INTRODUÇÃO}

Controladores lógicos programáveis (CLPs) estão presentes no controle de processos industriais há décadas [1]. Indústrias que querem automatizar sua produção utilizam os controladores lógicos programáveis para comandar os atuadores do processo. Os CLPs foram os grandes responsáveis pela revolução da indústria 3.0 [1]. Dessa forma, para os alunos de cursos de formação técnica que contemplem área de atuação em indústrias, por exemplo, Engenharia de Automação e Controle, precisam estar familiarizados com estes equipamentos já que atuarão com estes em seus futuros locais de trabalho.

Entretanto, a situação das universidades públicas com cortes de verbas acaba dificultando as melhorias de laboratórios [2]. Em específico, laboratórios de automação industrial, o aproveitamento do aprendizado por cada aluno seria maior se houvessem CLPs suficientes para todos. Com quantidades destes equipamentos insuficientes para a turma de alunos há maior esforço por parte dos docentes e discentes para que haja um aproveitamento do aprendizado sobre os controladores lógicos programáveis. Somente a teoria não é suficiente para a formação neste assunto.

Dois outros trabalhos em português foram encontrados na plataforma Periódicos da Capes, após pesquisa usando as palavras-chave "CLP" e "educacional". O trabalho de Castilho et al. [3] aborda o desenvolvimento de uma plataforma de produção automatizada, e usa um CLP comercial em parte do sistema. Um kit educacional para controle de nível foi produzido por Pereira et al. [4], que também utiliza um CLP comercial. Portanto, percebe-se na literatura a necessidade de construção de um CLP de baixo custo para aplicações educacionais.

Diante deste cenário, propõe-se uma medida para mitigar a falta de CLPs em laboratórios de Universidades: o desenvolvimento de um CLP de baixo custo. Inspirada na onda do "faça você mesmo" (DIY - Do It Yourself), a ideia é montar um CLP de baixo custo de modo que os alunos possam comprar os materiais e fabricar os seus próprios CLPs para as aulas ou ainda fazer com que o orçamento da instituição consiga adquirir CLPs pelo baixo custo de cada unidade comparado aos vendidos no mercado. Espera-se que com esta medida as aulas que precisem de CLPs para 29 parte prática fiquem mais proveitosas para os alunos. De forma que o aluno poderá até praticar em sua casa, já que ele terá o seu próprio CLP.

Este artigo está organizado em cinco seções. A Seção 2 descreve 0 dimensionamento dos circuitos que compõem o CLP proposto e o software utilizado para programação na linguagem Ladder. Na Seção 3 são apresentadas a placa de circuito impresso do CLP proposto e a interface do Ladder Maker para escrita da programação pelo usuário. A análise de custos entre o protótipo e três CLPs comerciais é feita na Seção 4. A Seção 5 apresenta as conclusões.

\section{MATERIAIS E MÉTODOS}

Para a construção de um controlador lógico programável, pode-se dividir o processo em duas vertentes: uma é a construção do hardware que engloba toda a estrutura física do equipamento, e a outra é a elaboração do software que corresponde ao sistema de programação que vai manipular o equipamento acionando e desligando suas entradas e saídas. Os detalhes de cada elaboração do software e hardware são apresentados a seguir.

\subsection{Hardware}

Analisando os CLPs mais utilizados na indústria e conferindo seus respectivos catálogos dos fabricantes, nota-se que eles possuem a seguinte configuração mínima: circuitos de entradas e saídas digitais (discretas) e analógicas, processador digital (CPU), memórias de programa (ROM) e de dados (RAM), canal de comunicação com um PC e fonte de alimentação elétrica [5]. Baseado nessa configuração mínima dos CLPs de mercado, foi planejada a configuração do hardware do CLP de baixo custo. Ele conterá:

- Tensão de operação em $24 V_{\mathrm{Cc}}$;

- 4 entradas de $24 \mathrm{~V}_{\mathrm{CC}}$;

- 4 saídas por contato seco;

- 2 entradas de 4 a $20 \mathrm{~mA}$;

- 2 saídas de 4 a $20 \mathrm{~mA}$.

Estas duas entradas e duas saídas de 4 a $20 \mathrm{~mA}$ foram concebidas devido ao padrão industrial de sensores e atuadores que trabalham nesta faixa de operação de corrente [6]. Para a 
integração destas entradas e saídas se faz necessário haver um controlador, que recebe as informações da programação do software de controle e atua na leitura ou escrita das portas de entrada e saída. Como a ideia é baratear o produto, foi escolhida uma plataforma de hardware que fosse open hardware e open software de forma que não haja custos com pagamento de licenças de uso. A plataforma escolhida foi o Arduino $@$, pela sua disponibilidade no mercado local e fácil utilização, pois se baseia no sistema plugand play, mas especificamente o ArduinoNano (c) que é uma das placas da plataforma [7].

\section{Alimentação do CLP}

A escolha da alimentação de $24 V_{C c}$ foi feita por ser um padrão industrial comum para sistemas de controle de extra baixa tensão [8]. Dessa forma, este CLP poderia ser incluído em algum teste de controle de processo industrial. Contudo, internamente há uma redução desta tensão para uma tensão de operação de $12 \mathrm{~V}_{\mathrm{Cc}} \mathrm{e}$ de $9 \mathrm{~V}_{\mathrm{cc}}$. A tensão de $12 \mathrm{~V}_{\mathrm{cc}}$ é utilizada para a operação das entradas e saídas de 4 a 20 mA. A tensão de $9 \mathrm{~V}_{\mathrm{CC}}$ é utilizada para alimentar a unidade de controle central e alimentar o circuito de saída a contato seco utilizando relés eletromecânicos.

A redução desta tensão de $24 \mathrm{~V}_{\mathrm{CC}}$ para $12 \mathrm{~V}_{\mathrm{CC}}$ e $9 \mathrm{~V}_{\mathrm{CC}}$ é realizada a partir de dois circuitos integrados (CI) reguladores de tensão, o CI 7812 e 7809. Estes CIs convertem a tensão de entrada em uma tensão de saída reduzida e regulada [9]. O problema destes CIs é o baixo rendimento, pois a diferença de tensão de entrada com a tensão de saída gera uma queda de potencial elétrico que é transformado em calor no circuito integrado, fazendo com que eles esquentem por seu próprio funcionamento. Poderia ter-se escolhido outra forma de reduzir a tensão de $24 \mathrm{~V}_{c c}$ para $12 \mathrm{~V}_{c c}$ e $9 \mathrm{~V}_{\mathrm{CC}}$, mas devido ao baixo custo de aquisição destes CIs eles foram as melhores opções de projeto. O circuito de entrada da alimentação pode ser visto na Figura 1.

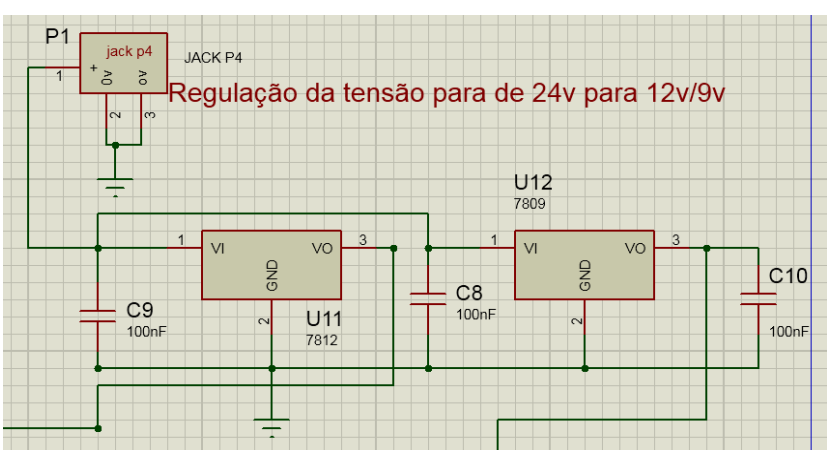

Figura 1: Circuito de alimentação do CLP. Fonte: O autor.

\section{Entradas de tensão 24 V cc}

O circuito de leitura de entrada de $24 V_{C C}$ é apresentado na Figura 2.

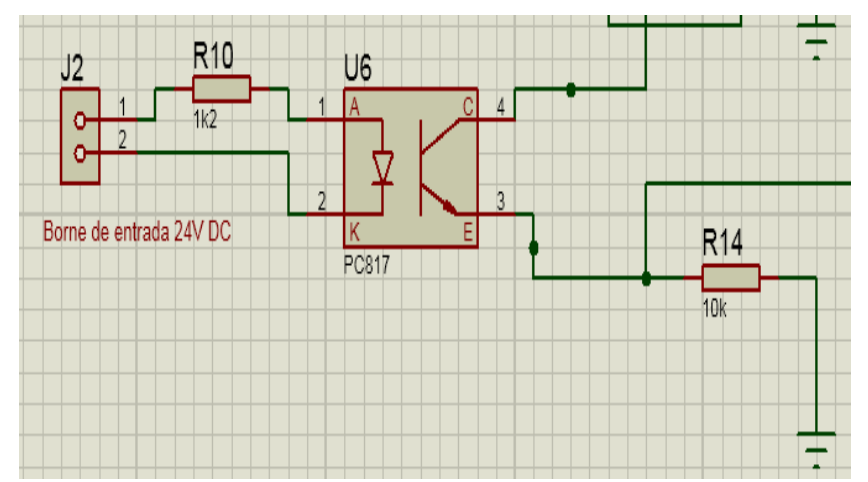

Figura 2: Circuito de leitura de tensão $24 \mathrm{~V}_{\mathrm{cc}}$.

Fonte: $\mathrm{O}$ autor.

O circuito é baseado na ideia da percepção de corrente elétrica por parte do controlador quando houver $24 \mathrm{~V}_{\mathrm{CC}}$ conectado à entrada do CLP. Há várias maneiras de montagem de um circuito eletrônico para este objetivo. Contudo, foi escolhida a utilização de um optoacoplador para esta interface. A escolha se deu de forma a garantir maior segurança ao circuito eletrônico do CLP, visto que o optoacoplador não possui contato elétrico entre seus circuitos de entrada e saída, de forma que há uma isolação óptica entre seus terminais.

Nos terminais de entrada do optoacoplador coloca-se um resistor para limitar a corrente oriunda da entrada $24 \mathrm{~V}_{\mathrm{CC}}$. Há diversos optoacopladores no mercado, pelo menor custo e fácil aquisição foi escolhida a utilização do EL817 que atende aos requisitos do projeto e possui queda de tensão de funcionamento no LED interno de 1,2 $\mathrm{V}_{\mathrm{CC}}[\mathbf{1 0}]$. Com entrada de $24 \mathrm{~V}_{\mathrm{CC}}$, corrente de entrada do optoacoplador de $20 \mathrm{~mA}$ e 
queda de tensão no LED interno ao optoacoplador de $1,2 V_{\mathrm{CC}} \circ$ cálculo do resistor ( $\mathrm{R} 10$ ) a ser utilizado se dá pela Equação (1):

$$
\mathrm{R} 10=\frac{24 \mathrm{~V}-1,2 \mathrm{~V}}{0,02 \mathrm{~A}}=1140 \Omega
$$

Para valores de tensão maiores e menores de $24 V_{c c} \circ$ circuito também irá funcionar, pois haverá uma passagem de corrente entre os terminais de entrada do optoacoplador. O EL817 possui uma corrente máxima de operação para o LED interno de $60 \mathrm{~mA}$, desta forma, na Equação (2) temos o valor máximo de tensão ( $V$ ) que pode-se colocar na entrada do CLP para que não haja queima do optoacoplador:

$$
\begin{array}{r}
\mathrm{V}_{\text {máx }}+1,2 \mathrm{~V}=\left(\begin{array}{c}
1140 \Omega \\
68,8 \mathrm{~V}
\end{array}\right. \\
0,06 \mathrm{~A}) \Rightarrow \underset{\text { máx }}{\text { (2) }}
\end{array}
$$

Para valores de entrada que não forneçam corrente suficiente para acionar o LED interno do optoacoplador, o controlador principal não irá detectar que há alguma tensão na entrada. Isto ocorrerá para uma corrente de entrada de $5 \mathrm{~mA}$ [8]. Pela Equação (3) encontramos o valor relativo da tensão em que o controlador não detectará leitura na entrada do CLP.

$$
\mathrm{V}_{\text {mín }}+1,2 \mathrm{~V}=\left(\begin{array}{c}
1140 \Omega \\
4,5 \mathrm{~V} .
\end{array} .0,005 \mathrm{~A}\right) \Rightarrow \underset{\text { mín }}{\text { (3) }}
$$

Nos terminais do fototransistor do EL817 temos conectado ao coletor uma tensão de $5 \mathrm{~V}_{\mathrm{CC}}$ oriunda do controlador e um resistor de pull-down ligado ao referencial do sistema. Por padrão, foi utilizado um resistor de pull-down de $1 \mathrm{k} \Omega$.

Ainda no terminal do emissor do fototransistor está ligado a uma entrada digital do circuito do microcontrolador do CLP. Assim que 0 fototransistor saturar, ou seja, o LED interno do optoacoplador estiver acionado, haverá uma passagem de corrente entre o coletor e emissor do fototransistor que por sequência também haverá uma corrente atravessando os terminais do resistor de pull-down, criando uma diferença de potencial em cima do resistor. Esta diferença de potencial será detectada pelo controlador, devido que uma das entradas do controlador está conectada ao resistor de pull-down. Com isto, o microcontrolador do CLP detecta se há ou não tensão na entrada do sistema pela leitura de nível lógico de tensão no resistor de pull-down do circuito de interface da entrada.

\section{Saídas a contato seco}

O circuito de saída à contato seco é apresentado na Figura 3.

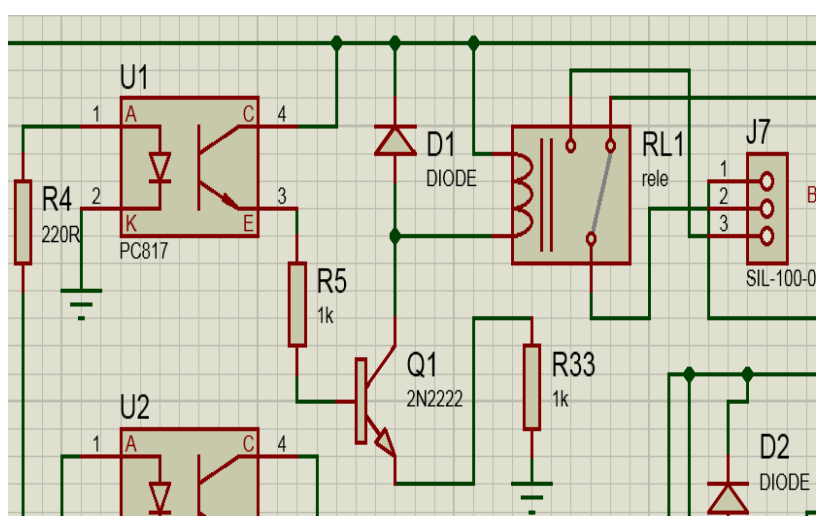

Figura 03: Circuito de saída de contato à relé. Fonte: $\mathrm{O}$ autor.

O princípio do circuito é o chaveamento da corrente que atravessa a bobina dos relés pela corrente oriunda do controlador principal para o LED do optoacoplador. Se o optoacoplador for acionado então o relé também é acionado, comutando seus contatos normalmente fechado (NF) e normalmente aberto(NA). Pelo motivo do circuito de leitura das entradas do CLP já utilizarem o optoacoplador EL817 então foi escolhido o mesmo para compor o circuito de acionamento das saídas.

Para fazer esta interface de chaveamento de controle utiliza-se de um transistor bipolar de junção NPN com terminal de base ligado ao terminal emissor do optoacoplador chaveado pela unidade central de controle. $O$ uso deste transistor se fazer necessário, pois os optoacopladores não possuem uma grande capacidade de condução de corrente [10]. Há uma gama de transistores que podem ser utilizados nesta aplicação. A família de transistores BCXXX consegue suprir esta necessidade. Desta forma, o transistor escolhido foi o BC337 pelo preço e facilidade de encontrar no comércio local [11]. O diodo colocado entre os terminais da bobina do relé é para a proteção do circuito quando ocorre a rápida comutação de corrente que atravessa a bobina originando uma tensão muito alta [12].

Para a fonte de alimentação deste circuito de saída foi utilizada a saída do CI 7809 que corresponde à $9 \mathrm{~V}_{\mathrm{CC}}$. A escolha foi baseada no uso de menor potência para o CLP. 
Como uma corrente de $5 \mathrm{~mA}$ é suficiente para saturar o transistor Q1 (vide Fig. 3) [11] e o valor fornecido pelo microcontrolador é de $5 \mathrm{~V}_{\mathrm{cc}}$, então - valor mínimo do resistor R5 é dado pela Equação (4):

$$
\mathrm{R} 5 \geq \frac{5 V-0,7 V}{0,005 A} \geq 860 \Omega .
$$

Consultando os manuais de fabricante de relés, percebe-se que correntes de $30 \mathrm{~mA}$ são suficientes para acioná-los [12]. Assim o valor deste resistor R33 é mostrado na Equação (5).

$$
\mathrm{R} 33 \leq \frac{9 V-5 V}{0,03 A} \leq 133,3 \Omega
$$

Também há um resistor R4 ligado ao terminal anodo do LED do optoacoplador. Este resistor limita a corrente fornecida pelo controlador central, impedindo que o LED do optoacoplador entre em falha. $O$ controlador fornece uma tensão de $5 \mathrm{~V}_{\text {CC }}$ e o LED do optoacoplador opera com corrente de $20 \mathrm{~mA}$, pela Equação (6) vemos o valor para este resistor.

$$
\mathrm{R} 4 \geq \frac{5 V-1,2 V}{0,02 A} \geq 190 \Omega \text {. }
$$

Este circuito possui formato de saída padrão de CLPs comerciais, usando contatos de um relé eletromecânico que podem ser usados em circuitos de corrente alternada. Desta forma, é possível controlar o acionamento de vários circuitos e equipamentos de maior potência como: circuitos de comando de motores elétricos, sessões de iluminação, máquinas industriais, autoclaves, dentre outros.

\section{Entradas analógicas de corrente de4 mA a $20 \mathrm{~mA}$}

Como é um padrão industrial para sensores e atuadores [8], foram incluídas entradas e saídas de corrente de $4 \mathrm{~mA}$ a $20 \mathrm{~mA}$. O circuito básico está esquematizado na Figura 4, e possui como elemento central o CI LM324.

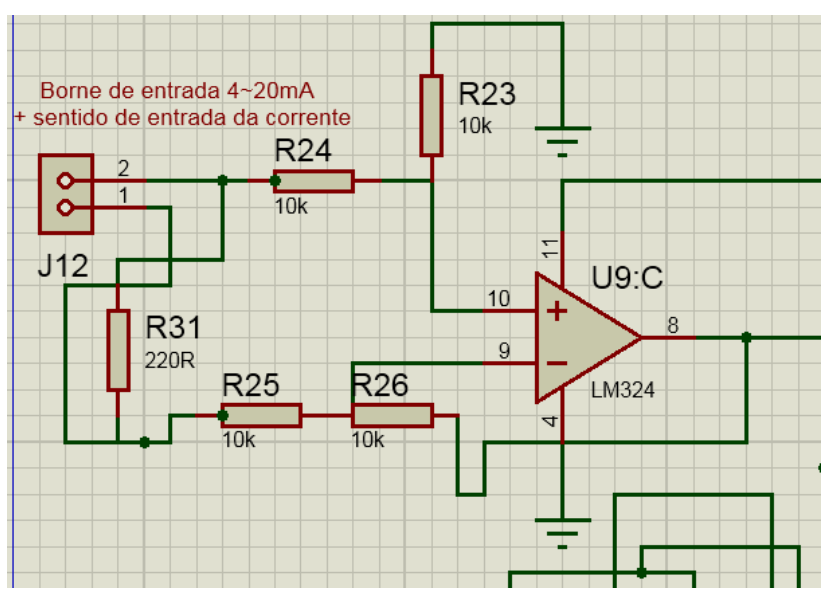

Figura 4: Circuito de entrada de $4 \mathrm{~mA}$ a $20 \mathrm{~mA}$. Fonte: $\mathrm{O}$ autor.

O princípio deste circuito da Figura 4 é o conceito de que uma corrente atravessando uma resistência elétrica gera uma diferença de potencial elétrico. Diante disto, pode-se saber a respectiva tensão elétrica gerada pela corrente de $4 \mathrm{~mA}$ a $20 \mathrm{~mA}$ nos terminais de entrada do CLP.

Um circuito com um resistor entre os terminais de entrada do CLP seria suficiente para captar a leitura de tensão nos terminais de entrada. Entretanto, o circuito de leitura do controlador lógico principal acaba que por interferindo na aferição da diferença de potencial, pois a corrente, que antes só atravessaria o resistor, também passaria a atravessar o controlador central por meio de seu circuito de leitura interno, algo que não é desejado visto que a leitura da tensão entre os terminais do resistor seria divergente da real corrente fornecida na entrada.

Desta forma, precisa-se colocar uma interface de isolação entre os terminais de entrada que recebem a corrente elétrica de $4 \mathrm{~mA}$ a $20 \mathrm{~mA}$ para os terminais de leitura internos do controlador central. Um circuito que consegue suprir esta necessidade é um amplificador operacional na configuração amplificador diferencial de ganho unitário, replicando na saída a diferença de potencial elétrico nos terminais de entrada [13].

A configuração dos resistores R23, R24, R25 e R26 na Fig. 4 conectados aos terminais positivo e negativo do amplificador operacional formam um circuito de ganho unitário. Desta forma, a diferença de potencial elétrico no resistor R31 será replicada no terminal de saída do amplificador operacional. A isolação deste tipo de circuito é 
dada pela elevada impedância entre os terminais de entrada do amplificador operacional [13].

Este circuito possui um detalhe técnico sobre o sentido de corrente na entrada do CLP. A tensão nos terminais do amplificador operacional deve estar de uma forma que o maior potencial esteja no terminal da entrada não-inversora do amplificador operacional e o menor potencial no terminal da entrada inversora, para que a tensão de saída seja positiva. Há a necessidade de que a tensão de saída do amplificador operacional seja positiva em relação ao referencial do circuito, pois o controlador central possui a especificação de leitura de tensões de 0 a $5 \mathrm{~V}_{\mathrm{CC}}$. Uma tensão negativa, oriunda da inversão do sentido da corrente acabaria por fornecer uma leitura errônea ao controlador central, já que o amplificador operacional saturaria em 0 [11]. Analisando a Fig. 4, nota-se que o sentido da corrente elétrica colocada na entrada do CLP precisa seguir 0 fluxo do Terminal 2 para $O$ Terminal 1.

O resistor R31 conectado aos terminais de entrada de $4 \mathrm{~mA}$ a $20 \mathrm{~mA}$ do CLP tem valor calculado na Equação (7), visto que a corrente especificada máxima fica em $20 \mathrm{~mA}$ e a tensão máxima de leitura é de $5 \mathrm{~V}_{\mathrm{Cc}}$ :

$$
\mathrm{R} 31=\frac{5 \mathrm{~V}}{0,02 \mathrm{~A}}=250 \Omega
$$

Os resistores do circuito de ganho unitário do amplificador operacional foram escolhidos para que haja a menor dissipação de potência com uma alta impedância. Para formar o ganho unitário basta que todos os quatro resistores sejam de mesmo valor. Há uma pequena diferença entre os valores dos resistores devido à faixa de tolerância, entretanto alta confiabilidade não é o foco do CLP de modo que este um problema da faixa de tolerância é aceitável ao projeto. O valor escolhido foi de $10 \mathrm{k} \Omega$.

Como o controlador central só permite leituras de 0 a $5 \mathrm{~V}_{\mathrm{CC}}$ em seus terminais, foi colocado um diodo zener de $5 \mathrm{~V}_{\mathrm{cc}}$ na saída do amplificador operacional para evitar que ocorra algum dano do circuito interno de leitura por algum eventual aumento da corrente de entrada do CLP.

O amplificador operacional escolhido foi o CI LM324 [14]. Este CI possui quatro amplificadores operacionais internos e não necessita de alimentação simétrica de forma que se encaixa na especificação da necessidade do circuito de leitura de entrada de $4 \mathrm{~mA}$ a $20 \mathrm{~mA}$. Do CI foram utilizados dois amplificadores operacionais para a entrada e dois foram utilizados para a saída de analógica, conforme será apresentado a seguir. Como a alimentação do CI é a mesma, a alimentação dos amplificadores operacionais é de $12 \mathrm{~V}_{\mathrm{cc}}$.

\section{Saídas analógicas de corrente de $4 \mathrm{~mA}$ a $20 \mathrm{~mA}$}

Assim como as entradas de leitura de corrente de $4 \mathrm{~mA}$ a $20 \mathrm{~mA}$ permitem que o CLP se comunique com sensores industriais, estas saídas permitem o controle de atuadores industriais. A Figura 5 mostra o circuito para as saídas analógicas.

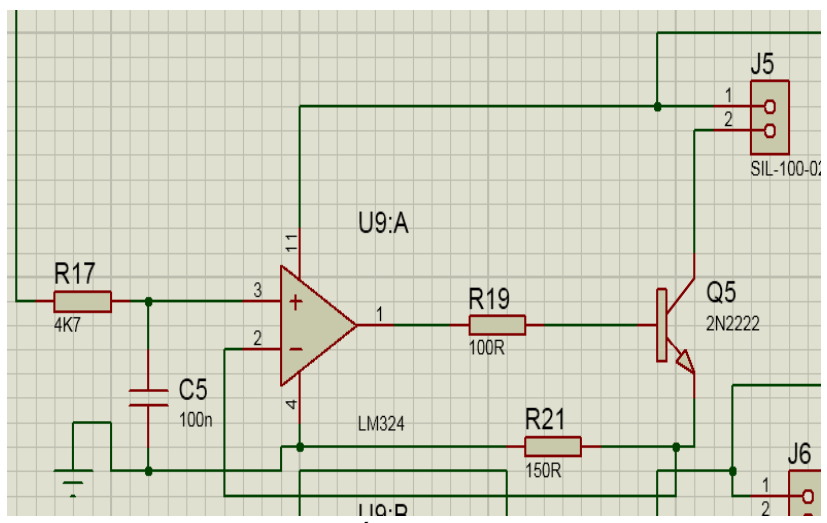

Figura 5: Circuito de saída de corrente.

Fonte: próprio autor.

Neste circuito, utiliza-se o conceito de seguidor de tensão utilizando amplificadores operacionais junto com um transistor de bipolar de junção. A tensão de saída $\left(V_{\text {saída }}\right)$ de um amplificador operacional é dada pela Equação (8), onde $A$ simboliza o ganho.

$$
\mathrm{V}_{\text {saída }}=A\left(V_{+}-V_{-}\right)
$$

Como os ganhos dos amplificadores operacionais são extremamente elevados [13], vê-se pela Equação (11) a tensão de entrada no terminal positivo é a mesma da entrada no terminal negativo.

$$
\begin{aligned}
& \frac{\mathrm{V}_{\text {saída }}}{A}=\left(V_{+}-V_{-}\right) \cong 0 ; \\
&\left(V_{+}-V_{-}\right) \cong 0 ;
\end{aligned}
$$




$$
V_{+} \cong V_{-}
$$

Diante desta particularidade, usa-se o artifício de colocar um resistor ligado à entrada do terminal negativo que também está ligado em realimentação negativa para que haja uma corrente constante fluindo pelo resistor R21. Esta corrente será dada seguindo a Lei de Ohm e será calculada para resultar em uma corrente de $4 \mathrm{~mA}$ a $20 \mathrm{~mA}$ de acordo com a entrada do terminal positivo do amplificador operacional pela Equação 12.

$$
\mathrm{I}_{\mathrm{R} 21}=\frac{V_{-}}{\mathrm{R} 21}
$$

Diante destas informações, percebe-se que uma carga colocada nos terminais de saída analógica do CLP não vai modificar a corrente que flui do coletor ao emissor do transistorQ5, pois o amplificador operacional estabiliza a corrente no resistor R21 pela tensão do terminal positivo mantendo uma corrente constante.

Para fazer o controle da tensão colocada sobre - terminal não-inversor do amplificador operacional, o controlador central usa uma modulação de pulsos (PWM - Pulse WidthModulation), visto que a diferença de potencial fornecida pelo controlador assume dois estados 0 ou $5 \mathrm{~V}_{\mathrm{CC}}$. Um circuito RC é utilizado para converter os pulsos oriundos do controlador central para um nível de tensão mais estável em uma faixa de valores de 0 a $5 \mathrm{~V}_{\mathrm{cc}}$. Pelas Equações (13) a (18), percebe-se que as tensões de entrada do amplificador operacional precisam variar entre os valores $0,6 \mathrm{~V}$ e $3 \mathrm{~V}$, para que a corrente de saída tenha valores de $4 \mathrm{~mA}$ a $20 \mathrm{~mA}$.

$$
\begin{aligned}
& \mathrm{I}_{\mathrm{R} 21(\text { máx })}=\frac{V_{-(\text {máx })}}{\mathrm{R} 21} ; \\
& 20 m A=\frac{V_{-}}{150 \Omega} ; \\
& V_{-(\text {máx })}=3 \mathrm{~V} ; \\
& \mathrm{I}_{\mathrm{R} 21(\text { min })}=\frac{V_{-(\text {mín })}}{\mathrm{R} 21} ; \\
& 4 m A=\frac{V_{-}}{150 \Omega} ; \\
& V_{-(\text {mín })}=0,6 \mathrm{~V} .
\end{aligned}
$$

O cálculo do circuito RC para essa faixa de valores é visto na Equação (19), analisando a impedância do capacitor.

$$
\begin{aligned}
& \text { Zcap }=\frac{1}{2 \pi f C} ; \\
& \text { Zcap } \cdot I+\mathrm{R}_{17} \cdot I=5 \mathrm{~V} .
\end{aligned}
$$

Para 3V máximo na entrada não inversora temos:

$$
\begin{aligned}
& 3 \mathrm{~V}=\frac{\mathrm{Z}_{\text {cap }}}{\mathrm{Zcap}+\mathrm{R} 17} \cdot 5 \mathrm{~V} ; \\
& 0,6 \mathrm{~V}=\frac{\mathrm{Z}_{\text {cap }}}{\mathrm{Zcap}+4,7 \mathrm{k} \Omega} ; \\
& 0,4\left(\mathrm{Z}_{\text {cap }}\right)=2820 \Omega ; \\
& \mathrm{Z}_{\text {cap }}=7050 \Omega ; \\
& 7050 \Omega=\frac{1}{2 \pi f C} .
\end{aligned}
$$

A frequência de $490 \mathrm{~Hz}$ é a frequência no terminal digital 9 do controlador central [15]. Porém, calcular um filtro para uma frequência muito próxima á frequência que se deseja atenuar faz com que o filtro não seja tão eficaz [15], assim foi escolhida a frequência de $300 \mathrm{~Hz}$. Temse:

$$
\begin{gathered}
7050 \Omega=\frac{1}{300 \cdot 2 \pi \cdot C} \\
C=75 n F .
\end{gathered}
$$

Desta forma, a partir de uma modulação PWM do controlador central consegue-se controlar a corrente de saída do CLP.

\section{Controlador Central}

O controlador central é a "inteligência" do CLP. É similar como o CPU de um computador. É por meio do controlador central que é possível programar o CLP proposto.

Para o controlador central do CLP de baixo custo foi utilizado o microcontrolador ATMEGA328P, mais especificamente o microcontrolador na placa da plataforma open hardware Arduino Nano(c [7]. A escolha do uso da plataforma foi pela sua disponibilidade de integração a projetos de controladores e pelo seu baixo custo no mercado comparado a outras 
plataformas, além de sua interatividade com o software de programação LadderMaker [17].

\subsection{Software}

Existe uma norma internacional chamada IEC 61131-3 que normatiza as linguagens de programação para os CLPs [16]. Como o intuito é a construção de um CLP mesmo que de baixo custo devemos seguir esta norma para que a assimilação do conteúdo por parte do aluno não seja deficitária em relação ao que o mercado de trabalho solicita.

A elaboração de um software de programação para um CLP envolve uma gama de dificuldades que não tem valor agregador ao projeto, visto que este aprendizado ficaria apenas para 0 programador do software. Além de que há um custo de pagamento de horas de programação a um programador para que o software fosse elaborado. Para diminuir o custo e facilitar a utilização do CLP por quaisquer aluno, foi-se buscado na internet $\mathrm{O}$ software chamado LadderMaker

[17].
O LadderMaker é um software de uso livre (open software). Não necessitando pagamento de licenças de uso. O software permite que o usuário programe o CI ATMEGA328P da placa Arduino Nano por meio da linguagem de programação Ladder [18, 19]. Dessa forma, O CLP desenvolvido está em conformidade com a IEC 61131-3 no que se refere à linguagem de programação Ladder.

\section{IMPLEMENTANDO 0 CONTROLADOR LÓGICO PROGRAMÁVEL}

A Figura 6 apresenta o circuito total do CLP desenhado no CAD Isis Proteus ${ }^{\odot}$. A definição dos pinos de entrada e saída do controlador central foi baseada pela especificação de escolha do software LadderMaker, pois o software vem com uma préconfiguração de funcionamento com os pinos de entrada e saída do Arduino $\mathrm{Nano}^{\odot}$, conforme Figura 7.

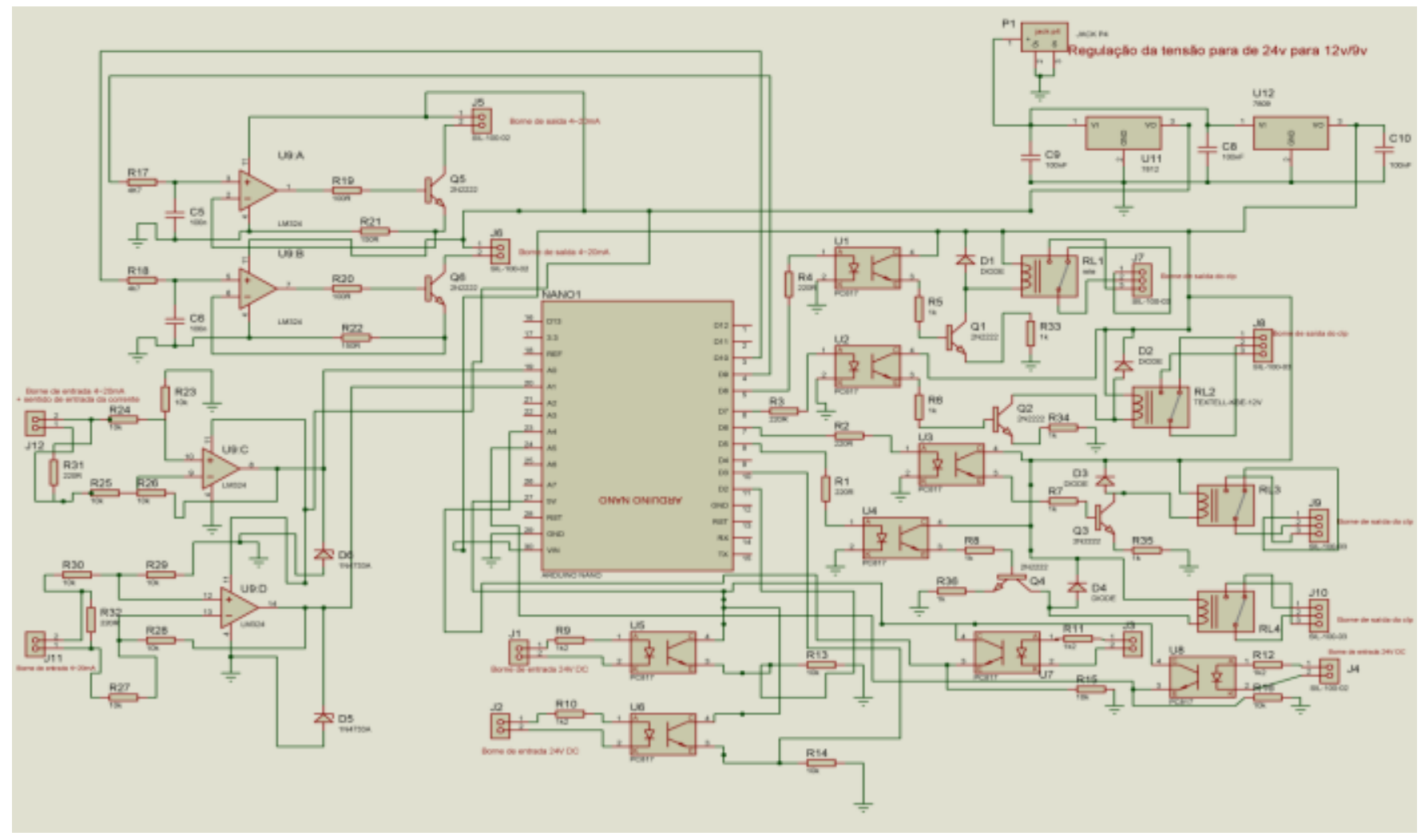

Figura 6: Circuito completo em CAD do CLP de baixo custo.

Fonte: Oautor. 


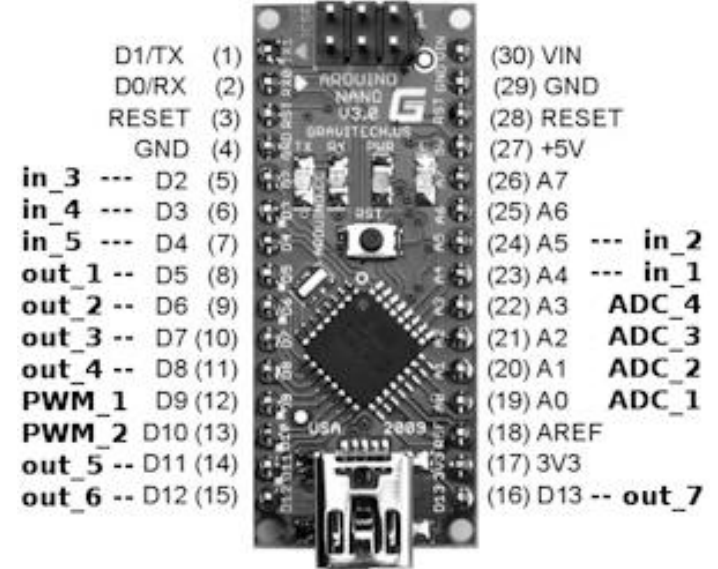

Figura 7: Pinos dedicados ao controlador pelo LadderMaker.

Fonte: [15].

\subsection{Diagrama esquemático para placa de circuito impresso}

Utilizando o software de desenho auxiliado por computador ISIS PROTHEUS ( ) foi feito o desenho da placa de circuito impresso para o controlador lógico programável de baixo custo. O desenho do circuito impresso foi realizado para permitir que os alunos pudessem ter a possibilidade de fabricar o seu próprio CLP de baixo custo.

A Figura 8 apresenta o desenho da placa de circuito impresso. Para a conexão do CLP com o mundo externo, seja por entrada ou por saídas foram utilizados, no desenho, bornes de conexão colocados em um mesmo lado da placa à ser confeccionada de modo que haja facilidade na conexão do cabeamento no CLP já que todos os bornes estão em um mesmo lado.

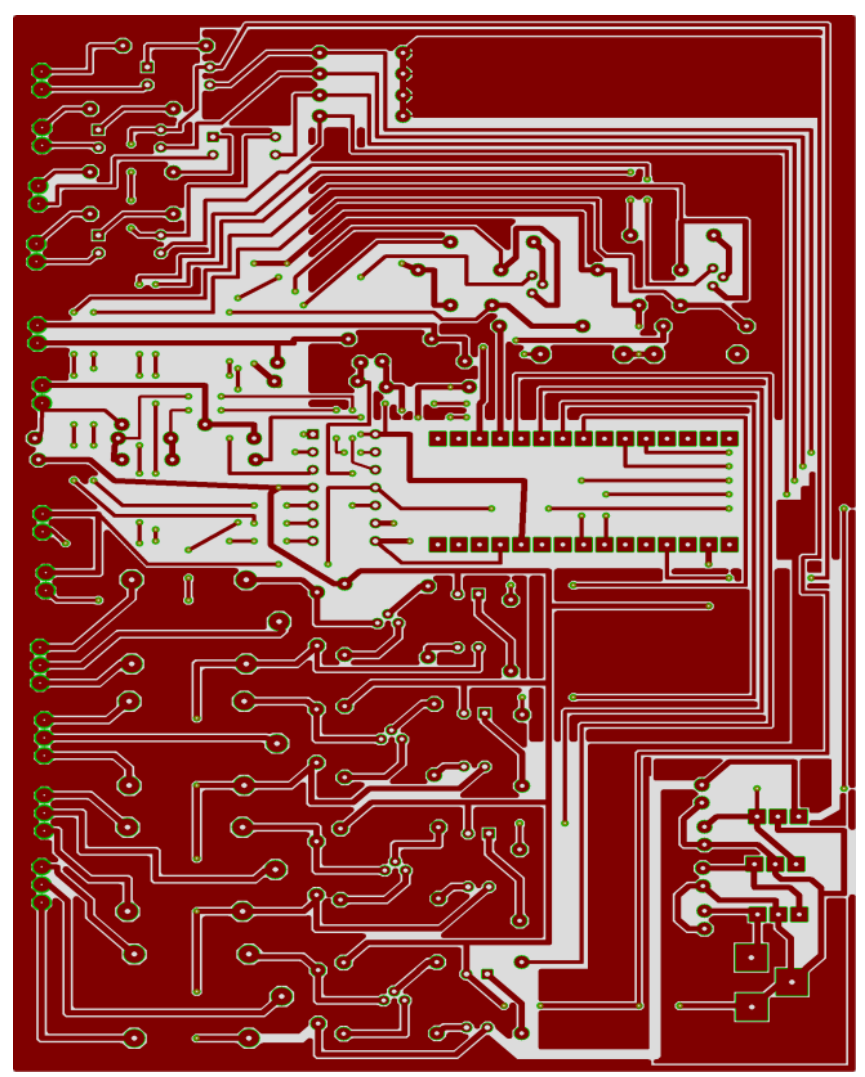

Figura 8: Desenho da placa de circuito impresso. Fonte: próprio autor.

\subsection{Manipulação do software LadderMaker}

O programa LadderMaker é distribuído gratuitamente na internet sob a licença GPL 3.0 [18]. Não é necessário fazer sua instalação. Contudo, até a data de elaboração deste documento, o software só estava disponível para sistemas operacionais Linux e Windows 7 . NaFigura 9 é mostrada a tela inicial do LadderMaker para a versão do sistema operacional Windows 7. 


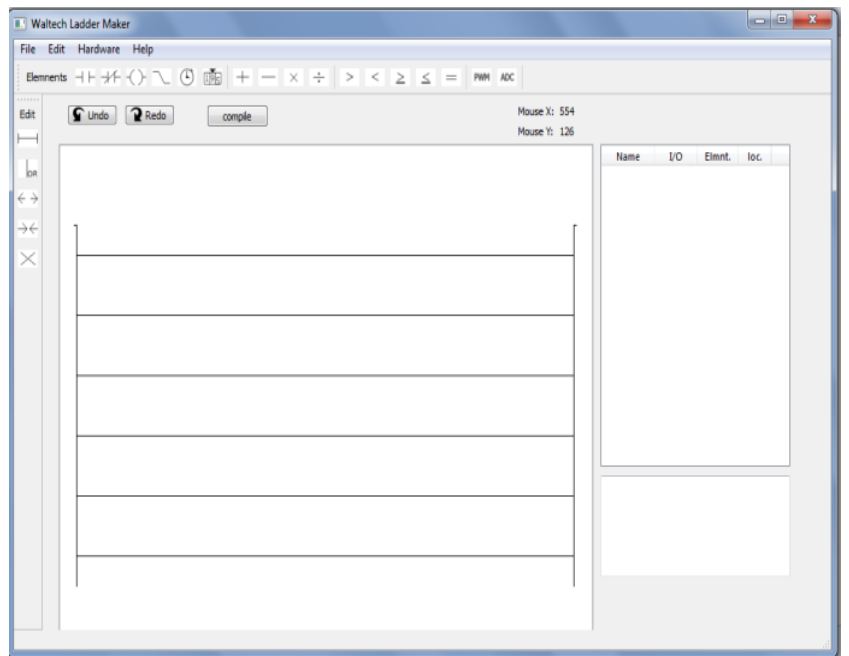

Figura 9: Tela inicial do LadderMake.

Fonte: $\mathrm{O}$ autor.

O programa permite utilizar as funções da linguagem de programação Ladder para programar as placas da plataforma Arduino(Uno, Nano e Mega). É possível utilizar as funções de contato aberto, contato fechado, contador, timer, operações aritméticas e lógicas, sinal em rampa,entrada e saída analógica, como também saída em pulso de onda modulada, tornando o uso do software bastante eficaz para uso didático. A Figura 10 apresenta algumas funções de entradas e saídas no LadderMaker.

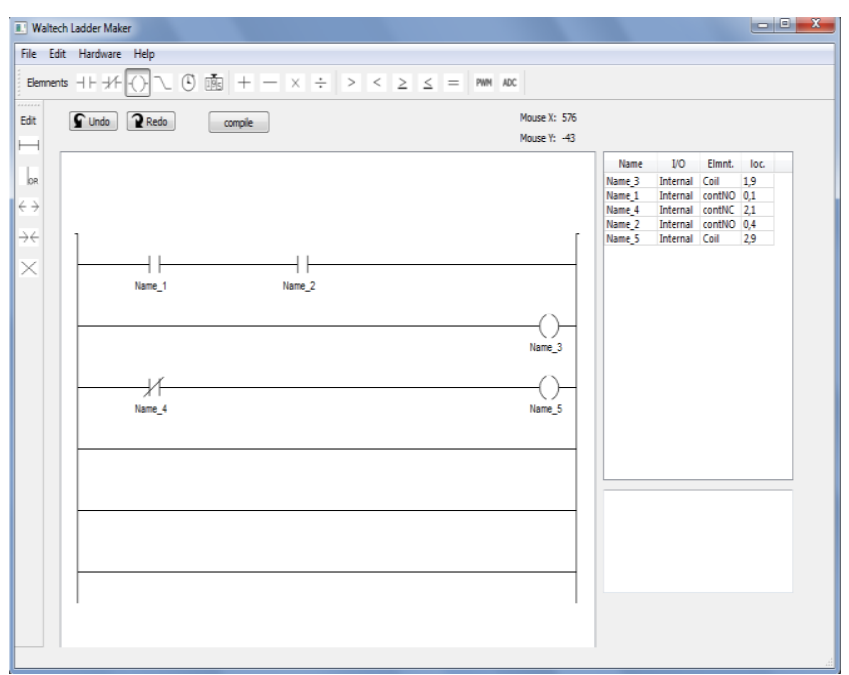

Figura 10: Exemplo de funções implementadas no software LadderMaker.

Fonte: O autor.

A simplicidade da interface de usuário do LadderMaker o torna uma boa escolha para compor a solução de um CLP de baixo custo para fins didáticos, onde o intuito é acelerar a curva de aprendizado dos estudantes.

\section{COMPARATIVO DE CUSTOS}

$\mathrm{Na}$ Tabela 1 encontra-se o quantitativo e o custo total dos materiais do projeto de construção do CLP didático proposto. Custando um valor médio menor do que $R \$ 100,00$ é possível montar um CLP para acompanhar as aulas práticas teóricas de disciplinas de estudos de automações industriais.

Tabela 1:Quantitativo de itens para confecção do CLP.

\begin{tabular}{|c|c|c|c|c|c|}
\hline ITEM & Quantidade & \multicolumn{2}{|c|}{ Preço unitário(médio) } & \multicolumn{2}{|c|}{ Preço total } \\
\hline Placa Arduino NANO & 1 & $R S$ & 30,00 & $R \$$ & 30,00 \\
\hline Capacitores 100nF & 6 & $\mathrm{RS}$ & 0,10 & $R \$$ & 0,60 \\
\hline Resistores & 36 & $\mathrm{R} \$$ & 0,10 & $R \$$ & 3,60 \\
\hline El817 & 8 & $\mathrm{RS}$ & 2,00 & $R \$$ & 16,00 \\
\hline LM324 & 1 & $R \$$ & 3,00 & $R \$$ & 3,00 \\
\hline LM7816 & 1 & $\mathrm{R} \$$ & 1,50 & $\mathrm{RS}$ & 1,50 \\
\hline LM7812 & 1 & $\mathrm{RS}$ & 1,50 & $R \$$ & 1,50 \\
\hline LM7809 & 1 & $R \$$ & 1,50 & $R \$$ & 1,50 \\
\hline Transistores BC337 & 6 & $\mathrm{RS}$ & 1,00 & $R \$$ & 6,00 \\
\hline $\ln 4007$ & 4 & $\mathrm{RS}$ & 1,00 & $R \$$ & 4,00 \\
\hline $\ln 4733$ & 2 & $\mathrm{R} \$$ & 2,00 & $\mathrm{R} \$$ & 4,00 \\
\hline Jack P4 & 1 & $\mathrm{RS}$ & 1,50 & $R \$$ & 1,50 \\
\hline Borne2vias & 8 & $\mathrm{RS}$ & 0,60 & $R \$$ & 4,80 \\
\hline Bornes 3 vias & 4 & $\mathrm{RS}$ & 0,80 & $R \$$ & 3,20 \\
\hline Placa fenolite $10 \times 15 \mathrm{~cm}$ & 1 & $\mathrm{R} \$$ & 6,00 & $R \$$ & 6,00 \\
\hline \multirow[t]{2}{*}{ Relé 5VDC } & 4 & $R \$$ & 2,00 & $R \$$ & 8,00 \\
\hline & & & & $R S$ & 95,20 \\
\hline
\end{tabular}

Fonte: O autor.

Para efeito de comparação, a Tabela 2exibe o valor de alguns modelos de CLP compactos de marcas atuantes no mercado [5], que são de porte comparado ao CLP didático. Percebe-se que há uma vantagem inicial financeira devido ao baixo custo do CLP aqui proposto. Salientando que o custo do projeto citado inclui somente os materiais para confecção, não incluindo custo de mão de obra e encargos financeiros. 
Tabela 2: Preços de compra de CLPs de modelo básico.

\begin{tabular}{|c|lr|}
\hline Controlador Lógico Programável & \multicolumn{2}{|c|}{$\begin{array}{c}\text { Custo do modelo } \\
\text { de entrada }\end{array}$} \\
\hline Siemens & R\$ & 800,00 \\
\hline WEG & $\mathrm{R} \$$ & 850,00 \\
\hline Rockwell & $\mathrm{R} \$$ & 650,00 \\
\hline
\end{tabular}

Fonte: O autor.

Ressalva-se que os custos foram listados para a construção de um protótipo funcional, que permite ao aluno realizar as práticas da aula de CLP. Na Figura 11, tem-se a foto do circuito montado em protoboard. A imagem apresenta o protótipo montado com uma placa Arduino Uno, por indisponibilidade de um Arduino Nano no momento da fotografia. Porém, esse fato não interfere na concepção do projeto, pois o Uno possui maior quantidade de terminais de entrada e saídas que apenas não foram utilizadas.

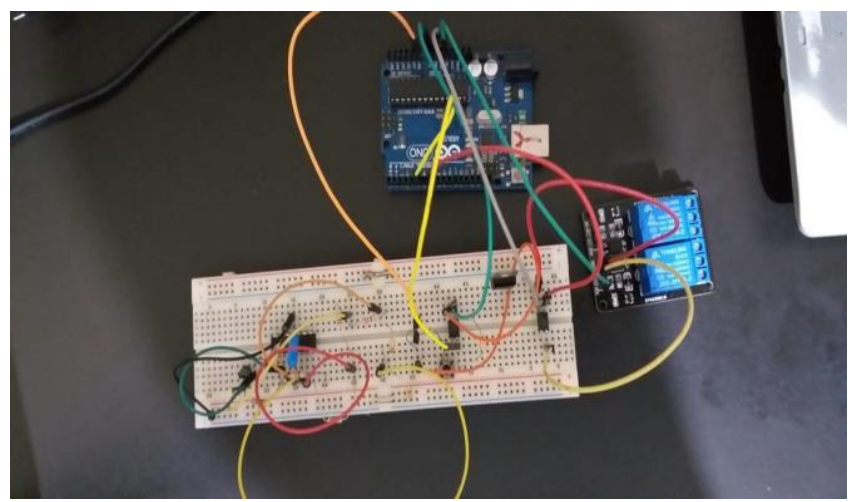

Figura 11: Circuito protótipo montado em protoboard. Fonte: $\mathrm{O}$ autor.

\section{CONCLUSÕES}

Nota-se uma diferença em entre o custo de confeç̧ão do CLP proposto neste trabalho eos preços de venda dos controladores mais básicos oferecidos pelas principais empresas de mercado.

As configurações de número de entradas e saídas e tipos de entrada e saída do CLP de baixo custo são similares aos equipamentos de mercado. Desta forma, as principais práticas de laboratório CLP poderão ser realizadas. A experiência dos alunos será mais interativa, pois os próprios alunos podem confeccionar seus CLPs, possibilitando que eles entendam mais profundamente as características de hardware, e consigam realizar diagnósticos sobre 0 equipamento e projetem novos componentes, habilidades que não são desenvolvidas quando existem CLPs prontos nos laboratórios. E a instituição de ensino também poderá adquirir mais CLPs permitindo práticas individuais e de melhor qualidade, aliadas com a teoria.

No tocante aos materiais utilizados, são materiais de fácil disponibilidade para compra no mercado local e sem necessidade de licença de uso de fabricantes. O software também segue essa linha de não necessidade de licença, o que facilita a obtenção e uso do mesmo pelos alunos. Assim, pode-se afirmar que o objetivo de confeccionar um CLP para laboratórios de práticas de automação industrial de baixo custo foi alcançado.

Nota-se, porém, que este CLP de baixo custo dificilmente será uma solução definitiva para o mercado. Em processos industriais, a confiabilidade dos equipamentos deve ser alta e um CLP deve acompanhar este mesmo critério, tanto por seus componentes quanto por sua confecção.

Os próximos passos serão dedicados a tornar a solução mais completa, montando a placa em um gabinete apropriado e colocando adesivos de identificação das entradas e saídas e um canal de comunicação via rede RS-485, tornando um produto mais profissional para as exigências do mercado educacional.

\section{REFERÊNCIAS}

[1] SILVA, R. R. da. HISTÓRIA DO CLP. In: SILVA, R. R. da. Blog Automação Industrial, 21 nov. 2012. Disponível em:

https://goo.gl/msdGvd. Acesso em: 10 out. 2018.

[2] OLIVEIRA, C. Universidades Federais fecham laboratórios de cursos. In: PRAGMATISMO POLÍTICO, 31 ago. 2017. Disponível em: https://goo.gl/yLhxni. Acesso em: 10 out. 2018.

[3] CASTILHO, R. A. et al. Apresentação de métodos para implementação de plataformas colaborativas de Sistemas Automatizados de Produção. Revista Gestão da Produção, Operações e Sistemas, Bauru, Ano 7, n. 2, p. 81-95, 2012.

DOI: https://doi.org/10.15675/gepros.v0i2.561. Disponível em:

https://revista.feb.unesp.br/index.php/gepro s/article/view/561. 
[4] PEREIRA, J. P. P.; VALENTIM, R. A. M.; CASTRO, B. P. S. Kit Educacional para Controle e Supervisão Aplicado a Nível. Holos, Ano 25, v. 2, p.68-72, 2009.

[5] GOOGLE SHOPPING. CLPs básicos. Disponível em: https://goo.gl/seMDy3. Acesso em: 11 out. 2018.

[6] FREITAS, C. M. Controladores Lógico Programáveis - CLP - Parte 3. In: EMBARCADOS. $11 \mathrm{fev}$. 2014. Disponível em:

https://goo.gl/KXnD3r. Acesso em: 11 out. 2018.

[7] GUIA ARDUINO NANO. In: ARDUINO.

Disponível em: https://goo.gl/Kz9STc. Acesso

em: 11 out. 2018.

[8] CASSIOLATO, C. Redes Industriais. In: SMAR TECHNOLOGY COMPANY, c2019. Disponível em: https://goo.gl/PnQJcv. Acesso em: 11 out. 2018.

[9] KEC. Datasheet CI 7805. Coréia. Revisão 1. 2010. 20p.

[10] EVERLIGHT. EL817. Disponível em: https://goo.gl/oGZLWK. Acesso em: 11 out. 2018.

[11] ONSEMI. Datasheet BC337. Colorado, EUA. Revisão 8. Nov. 2013. 5p.

[12] BRAGA, N. C. Como funcionam os relés. Instituto NCB. Disponível em:

https://goo.gl/qdUANz. Acesso em: 18 out. 2018.

[13] SACCO, F. Buffers e Seguidores de tensão. Disponível em: https://goo.gl/a4eaVv. Acesso em: 18 out. 2018.

[14] TEXAS INSTRUMENTS. Datasheet LM324. Texas, EUA, 2015.

[15] FRITZEN, C. Criando uma saída analógica de 0 a $5 \mathrm{~V}$ com arduino. Disponível em:

https://goo.gl/Bu6aTw. Acesso em: 18 out. 2018.

[16] HEINZ, J.; TIEGELKAMP, M. IEC 61131-3:

Programming Industrial Automation

Systems. Alemanha: Springer-Verlag, 2001. 240p.

[17] LADDERMAKER. Laboratório de Garagem. Disponível em: https://goo.gl/LeMsX8. Acesso em: 18 out. 2018.

[18] PETRUZELLA, F. D. Fundamentos do desenvolvimento de diagramas e programas em lógica ladder para o CLP, em
Controladores Lógico Programáveis, 4. ed. Mc Graw Hill Education, 2014.

[19] BOLTON, B. Programmable Logic Controllers, 4. ed. Newnes, 2006. (Capítulo 4 Ladder and functional block programming) 